\title{
Geschlechterrepräsentation in Nachrichtentexten
}

\author{
Der Einfluss von geschlechterbezogenen Sprachformen und Fallbeispielen auf \\ den gedanklichen Einbezug von Frauen und die Bewertung der Beitragsqualität
}

Christoph Klimmt / Verena Pompetzki / Christopher Blake

\begin{abstract}
Zwei Experimente $(N=41$ und $N=67)$ prüfen, inwiefern die Gleichbehandlung der Geschlechter in einem Zeitungsartikel in Bezug auf die sprachliche Formulierung (Schrägstrich-Schreibweise, z. B. „Die Beamt/inn/en“, im Vergleich zum generischen Maskulinum) und in Bezug auf die Zusammenstellung zitierter Fallbeispiele (eine weibliche und eine männliche Person als Fallbeispiele im Vergleich zu ausschließlich männlichen Personen als Fallbeispiele) zu einem veränderten gedanklichen Einbezug von Frauen seitens der Rezipient/inn/en fübren. Beide Gestaltungsfaktoren haben einen relevanten Einfluss auf die gedankliche Berücksichtigung von Frauen. Zugleich zeigt sich jedoch, dass die Schrägstrich-Schreibweise mehr Lesezeit verlangt und von den Rezipient/inn/ en hinsichtlich der journalistisch-sprachlichen Qualität schlechter bewertet wird. Implikationen für die genderbezogene journalistische Praxis und die weitere genderbezogene Nachrichtenrezeptionsforschung werden diskutiert.
\end{abstract}

Schlagwörter: Zeitung, Rezeption, Geschlecht, Geschlechterdarstellung, Einbezug von Frauen, Fallbeispiel, Gleichberechtigung, Medienwirkung, Situationsmodell, Experiment

\section{Problemstellung}

Die Darstellung der Geschlechter in massenmedialen Botschaften ist bereits seit den 1970er Jahren Gegenstand kommunikationswissenschaftlicher Forschung (z. B. Bate, 1978; Martyna, 1978; Purnell, 1978; zur linguistischen Diskussion: Pusch, 1979; Trömel-Plötz, 1982). Dabei steht die Frage nach der angemessenen, gleichberechtigten Repräsentation der Geschlechter weit oben auf der Agenda, weil geschlechtsbezogene soziale Ungleichheiten in den meisten modernen Gesellschaften bislang nicht ausgeräumt werden konnten. Aus der Sicht der feministischen Kommunikationsforschung ist die mediale Repräsentation der Geschlechter daher Ausdruck und Dimension bestehender gesamtgesellschaftlicher Machtgefüge und -konflikte (z. B. Purnell, 1978; Mucchi-Faina, 2005, zur konzeptuellen Fundierung: Carroll, 1956). Vor diesem Hintergrund ergibt sich die Frage, wie Medien mit den real existierenden Gender-Ungleichheiten umgehen und ob sie durch bestimmte Verhaltensweisen zu deren Fortbestand oder ihrer Überwindung beitragen können (z. B. Ganahl et al., 2003).

Geschlechterdarstellungen kommen in den Massenmedien in vielen Varianten vor. Mit Blick auf Nachrichtenmedien sind zunächst sprachliche Repräsentationen der Geschlechter in Textbotschaften zentral, wie sie in Zeitungen, Zeitschriften oder im Internet verbreitet werden. Im deutschsprachigen Raum steht dabei der traditionelle Gebrauch des generischen Maskulinums im Mittelpunkt, also die Verwendung rein männlicher Formen für Sammelkategorien, die inhaltlich Männer und Frauen einschließen sollen (z. B. „die Beamten“; vgl. Braun et al., 2005). Diese Darstellungsform steht jedoch in der Kritik, die gesellschaftliche Benachteiligung von Frauen zu reflektieren und sogar $\mathrm{zu}$ zementieren. Auch deshalb wurden mittlerweile verschiedene alternative Formen 
der Geschlechtsrepräsentation vorgeschlagen, die die konsequente formale Berücksichtigung beider Geschlechter in der Sprachgestaltung ermöglichen. Beispiele hierfür sind neue Schreibformen wie „BeamtInnen“ oder die Nutzung von Schrägstrichen innerhalb von Wörtern, mit denen beide Geschlechter innerhalb der Buchstabenfolge erkennbar werden sollen (z. B. „Die Beamt/inn/en“). Wissenschaftliche Befunde (z. B. Stahlberg \& Sczesny, 2001) deuten darauf hin, dass sprachliche Gestaltungen, die explizit beide Geschlechter erwähnen, den gedanklichen Einbezug von Frauen bei Leser/inne/n steigern. Andererseits stehen eben solche Formen immer wieder in der Kritik, die Sprachqualität, Sprachästhetik und sogar die Lesbarkeit von (journalistischen) Texten zu torpedieren (vgl. hierzu Döring, 2003; Rothmund \& Christmann, 2002) oder die Lesezeit zu verlängern (Irmen \& Kaczmarek, 2000). Der Widerstand gegen Änderungen der Sprachpraxis ist - auch gestützt auf die genannten Argumente - erheblich (z. B. Penelope, 1982; Parks \& Roberton, 2000).

Darüber hinaus ist im vorliegenden Kontext die Frage nach der angemessenen Berücksichtigung beider Geschlechter auf der Ebene der (selektiven, beispielhaften) medialen Darstellung sozialer Realität von Bedeutung. Hier ist insbesondere auf den Anteil der Frauen und Männer an der Gesamtheit der in den Nachrichten individuell vorgestellten Personen abzuheben. Denn von solchen Fallbeispielen gehen erhebliche Einflüsse auf die Rekonstruktion journalistisch berichteter Ereignisse durch die Rezipient/ inn/en aus (z. B. Zillmann \& Brosius, 2000). Zwar liegen bislang zu den Folgen des Geschlechts der im Fallbeispiel erwähnten Person(en) auf den gedanklichen Einbezug von Frauen bei der Rezeption keine empirischen Erkenntnisse vor, doch liegt analog zur Sprachebene die Vermutung nahe, dass eine (stärkere) Berücksichtigung weiblicher Personen im Kreis der präsentierten Fallbeispiele den gedanklichen Einbezug von Frauen durch die Rezipient/inn/en verstärken sollte.

Der vorliegende Beitrag geht diesen Überlegungen empirisch nach und untersucht den Einfluss der journalistischen Darstellung der Geschlechter (in der Sprache und in Fallbeispielen) auf den gedanklichen Einbezug von Frauen beim (Nachrichten-)Publikum. Zugleich wird die Frage thematisiert, wie das Publikum die journalistisch-sprachliche Qualität von Berichten mit gleichberechtigten Schreibweisen bewertet.

\section{Theoretische Grundlagen}

\subsection{Sprachliche Geschlechterrepräsentation und Textverstehen}

Die Rezeption medialer Texte (einschließlich auditiver und audiovisueller Medienbotschaften) wird in der Kommunikationswissenschaft unter Rückgriff auf kognitionspsychologische Modelle rekonstruiert. Textverstehen betrifft die mentale Repräsentation der Objekte, Personen, Handlungen und Ereignisse, die ein Text beschreibt. Um etwa eine Zeitungsmeldung über eine Demonstration zu verstehen, ist es erforderlich, sich eine innere Vorstellung von Ort, Zeit, Anlass, Identität und Anzahl der agierenden Personen aufzubauen und wechselseitige Bezüge zwischen diesen Elementen (sowie zu Wissensbeständen außerhalb des eigentlichen Berichts, etwa des demonstrationsrelevanten Politikfelds) herzustellen. Diese Form integrierter mentaler Repräsentation von Sachverhalten wird als Situationsmodell bezeichnet (Zwaan \& Radvansky, 1998). Situationsmodelle entstehen während der Medienrezeption, indem Sachinformationen aus dem Text extrahiert und organisiert werden, zugleich aber auch eigenes Vorwissen abgerufen wird, um (noch) vorhandene Repräsentationslücken zu schließen oder die eigene Sachverhaltsvorstellung anzureichern (Kintsch, 1988). Situationsmodelle 
werden kontinuierlich aktualisiert, indem neue textbasierte („bottom-up“) und/oder vorwissensbasierte („top-down“) Informationen berücksichtigt werden. Die mentale Repräsentation von textlich beschriebenen Begebenheiten gestaltet sich demnach hochgradig individuell, weil persönliches Vorwissen mit den aus dem Text entnommenen Informationen integriert wird. Da erhebliche individuelle Unterschiede im Vorwissen anzunehmen sind, lässt sich die Beschaffenheit von Situationsmodellen allein aus den im Text explizit enthaltenen Informationen nur begrenzt vorhersagen.

Vor diesem Hintergrund ist die teilweise stark normativ aufgeladene Debatte zur sprachlichen Gleichbehandlung der Geschlechter in Texten differenzierter zu betrachten. Denn aufgrund des erheblichen Einflusses vorwissensbasierter top-down-Prozesse kann nicht davon ausgegangen werden, dass eine konsequente und gut sichtbare sprachliche Gleichbehandlung der Geschlechter zu einer substanziellen Verbesserung des gedanklichen Einbezugs beider Geschlechter beim Publikum führen muss. Vielmehr entscheiden geschlechtsbezogene Vorwissensbestände mit darüber, ob ein Situationsmodell eine angemessene Geschlechterrepräsentation erhält oder ob Verzerrungen auf dieser Dimension gegenüber der Textintention bzw. der dem Text zugrunde liegenden realen Begebenheit auftreten.

Andererseits ist aber natürlich auch in Rechnung zu stellen, dass sprachlich codierte Detailinformationen sehr wohl Eingang in mentale Modelle finden (können), und zwar mit umso größerer Wahrscheinlichkeit, je häufiger sie im Text enthalten sind. Denn die bottom-up-Prozesse der Konstruktion von Situationsmodellen erfassen zwangsläufig eher solche Informationen, die gut sichtbar und/oder stabil während des Rezeptionsvorgangs verfügbar sind. Ein Text, der beide Geschlechter an jeder möglichen Stelle explizit erwähnt, hat daher durchaus theoretisch begründbare Chancen, zu einer anderen Geschlechterberücksichtigung (das heißt in der Regel zu einem angemesseneren gedanklichen Einbezug von Frauen) im Situationsmodell der Leser/innen zu führen als ein Text, der immer nur ein Geschlecht (in der Regel das generische Maskulinum) explizit erwähnt (Irmen \& Linner, 2005). In der Sozialpsychologie und in der Linguistik sind verschiedene Studien vorgelegt worden, die diese Annahme empirisch untermauern (Braun et al., 1998, 2005; Irmen \& Köhncke, 1996; Irmen \& Roßberg, 2004; Scheele \& Gauler, 1993; Stahlberg \& Sczesny, 2001).

Ein zweites gewichtiges Argument für die potenzielle Wirksamkeit sprachlicher Gleichbehandlungen der Geschlechter (im Sinne eines verbesserten gedanklichen Einbezugs beider Geschlechter durch das Publikum) bezieht sich gerade auf das für Situationsmodelle so wichtige Vorwissen. Denn angesichts der großen Menge medialer Texte, die in modernen Informationsgesellschaften rezipiert werden, kommt der dominanten Art der sprachlichen Geschlechterrepräsentation eine vorwissensprägende Funktion zu. Wer also über Jahrzehnte Bücher, Zeitungsberichte und andere Texte gelesen hat, in denen hauptsächlich das generische Maskulinum verwendet wurde, dürfte Vorwissensstrukturen angelegt haben (vgl. etwa Roskos-Ewoldsen et al., 2004, die eine Verbindung zwischen Situationsmodellen und der Kultivationsforschung entwickeln), die das männliche Geschlecht bevorzugen. Beispielsweise dürften derartig vorgeprägte Personen beim Aufbau eines Situationsmodells zu einem unbekannten Text zunächst davon ausgehen, dass eine nicht näher beschriebene Person männlich ist oder dass sich hinter genusneutralen Termini wie „die Abgeordneten“ eher Männer als Frauen verbergen dürften (vgl. dazu auch Döring, 2003; Irmen \& Linner, 2005; Prentice, 1994). Insofern kommt der konsequent bei allen Personen- und Gruppennennungen explizit angewendeten Gleichbehandlung der Geschlechter auch und gerade eine langfristige Wirkung auf genderbezogene Vorwissensstrukturen zu. Diese sollte sich dann wiederum bei der 
späteren Lektüre unterschiedlicher Texte positiv im Sinne eines verbesserten Einbezugs beider Geschlechter bemerkbar machen.

\subsection{Fallbeispiele und die (genderbezogene) mentale Repräsentation medial berichteter Sachverhalte}

In Anlehnung an Daschmann (2001, S. 79) werden Fallbeispiele als mediale in Form von Zitaten oder Schilderungen vermittelte Einzelfallinformationen definiert. Ihre Präsentation dient dazu, eine über den Einzelfall hinausgehende Aussage über die Wahrscheinlichkeit oder Häufigkeit eines in der Realität existierenden Merkmals oder Sachverhalts $\mathrm{zu}$ treffen. Verschiedene Studien haben ergeben, dass Fallbeispiele das Publikum auf mehreren Ebenen beeinflussen. Sie können sich sowohl auf die Vorstellungen (kognitive Effekte) als auch die Einstellungen und Meinungen (persuasive Effekte) von Rezipienten zu einem Sachverhalt mitunter gravierend auswirken (Daschmann, 2001).

Kognitive Effekte sind in der bisherigen Forschung am besten dokumentiert. So berichten Zillmann, Perkins und Sundar (1992), dass die Einschätzung über die Wahrscheinlichkeit, nach einer anfangs erfolgreichen Diät wieder an Gewicht zuzunehmen, von Leser/inne/n eines Stimulusartikels entsprechend des relativen Anteils jener Fallbeispiele im Artikel beeinflusst wurde, die einen solchen ,Jojo'-Effekt berichteten. Diese Wirkung zeigte sich trotz einer in allen Varianten des Texts genannten statistischen Information über die tatsächliche Prävalenz von ,Jojo'-Effekten. Zahlreiche weitere Studien ergaben vergleichbare Fallbeispiel-Effekte (z. B. Gibson \& Zillmann, 1994; Daschmann, 2001).

Aktuelle Ansätze erklären den starken Einfluss von Fallbeispielen auf die Realitätsvorstellungen von Rezipient/inn/en mit der menschlichen Lern- und Entwicklungsgeschichte. So argumentieren Brosius, Schweiger und Rossmann (2000), dass dem Menschen in der Alltagskommunikation in der Regel nur auf Einzelfällen basierende Informationen zur Verfügung stehen, um sich Urteile über ein bestimmtes Thema oder Problem zu bilden. Die Meinungen von Bekannten, die Erlebnisse von Freund/inn/en oder die Erfahrungen der Nachbar/inne/n seien seit jeher die hauptsächliche Informationsquelle. Lernen und Urteilen auf Basis von Einzelfällen lässt sich dementsprechend als „eine über Jahrhunderte gewachsene menschliche Konstante“ beschreiben (Brosius et al., 2000, S. 172; vgl. auch Daschmann, 2001).

Von Interesse ist im Rahmen der hier vorliegenden Problemstellung nicht der bisher in Studien zur Fallbeispielwirkung untersuchte Grad der Übernahme von aussagebezogenen Realitätsvorstellungen aus Fallbeispielen. Vielmehr stellt sich die Frage, ob sich die Verteilung des Geschlechts der Urheber von Fallbeispielen in der vom Publikum wahrgenommenen Geschlechterzusammensetzung der Gruppe niederschlägt, für die die Fallbeispiele stehen (sollen). Allgemeiner ausgedrückt geht es darum zu ergründen, ob Fallbeispiele auch zur Generalisierung von aussagenunabhängigen Merkmalen ihrer Urheber/innen auf die jeweilige Grundgesamtheit führen, aus der sie stammen. In diesem Fall müssten die Leser/innen von Texten, in denen gleichermaßen Frauen und Männer als Fallbeispiele präsentiert werden, den Anteil von Frauen, die am berichteten Geschehen teilnehmen, höher einschätzen als Leser/innen von Texten, in denen ausschließlich männliche Fallbeispiele vorkommen.

Da Fallbeispiele ein weit verbreitetes journalistisches Stilmittel sind, könnte eine geschlechterbezogen ausgewogene Fallbeispielpräsentation neben der Verwendung einer gleichberechtigten Schreibweise also eine weitere Möglichkeit für Journalist/inn/en darstellen, einen angemessenen mentalen Einbezug von Frauen während der Rezep- 
tion zu fördern. Dieser Effekt wäre aus journalistisch-praktischer Perspektive ebenso bedeutsam wie der oben diskutierte Aspekt der sprachlichen Geschlechterrepräsentation, weil Journalist/inn/en hier ebenfalls (wenn nicht sogar noch mehr) Wahlfreiheit bei der Ausgestaltung ihrer Texte und Beiträge besitzen: Wen sie als Fallbeispiel vorstellen, bleibt ihnen in der Regel überlassen, so dass ein empirisch nachweisbarer Effekt der Geschlechterzusammensetzung von Fallbeispielen Implikationen für die genderbezogene Alltagspraxis journalistischen Handelns besitzen würde.

\subsection{Lesbarkeit und Lesbarkeitsurteile in Abhängigkeit genderbezogener Schreibweisen}

Der häufig (z. B. von der UNESCO: Hellinger \& Bierbach, 1993) geäußerten Forderung nach gender-sensitiver Textgestaltung und speziell nach sprachlicher Geschlechtergleichbehandlung steht eine eklatant geringe Bereitschaft vieler Autor/inn/en gegenüber, solche Formen in ihre journalistischen, wissenschaftlichen oder anderen Texte zu integrieren. Parks und Roberton (1998) berichten von deutlich negativen Einstellungen in einem Befragungssample gegenüber einer „non-sexist language“. Die Zurückweisung einer sprachlichen Gleichbehandlung der Geschlechter begründen viele Befragte der Studie mit den Argumenten und Behauptungen, die Blaubergs (1980) als typische Muster des Widerstands gegen eine sprachliche Geschlechtergleichbehandlung zusammengestellt hat. Dazu gehören beispielsweise die Trivialisierung des Sprachgebrauchs als irrelevant für die gesellschaftliche Gender-Debatte oder der Zensurvorwurf an Befürworter einer geschlechtergleichbehandelnden Sprache. Ein weiteres Argument der Liste von Blaubergs (1980), das auch in der Studie von Parks und Roberton (1998) empirische Substanz aufweist, ist der Aufwand, der mit einer Umgewöhnung der Bevölkerung an neue Sprachregeln verbunden sei. Dieser Mehraufwand bezieht sich sowohl auf die Sprachproduktion (Schreiben, Sprechen) als auch auf die Sprachrezeption (Lesen, $\mathrm{Zu}$ hören). Döring (2003, S. 28) zitiert das in diesem Kontext häufig verwendete Argument von Autor/inn/en: „Aus Gründen der besseren Lesbarkeit wird im Text nur die männliche Form verwendet."

Gerade für journalistische Texte ist eine positive Sprachanmutung und flüssige Rezipierbarkeit essenziell, weil - anders etwa als behördliche Amtssprache - Journalist/ inn/en um die Lektürebereitschaft ihres Publikums werben wollen und werben müssen. Doch auch im Kontext der Amtssprache wird nach Lösungen gesucht, die die gesetzlichen Maßgaben zur (sprachlichen) Gleichbehandlung der Geschlechter mit einer zufriedenstellenden Lesbarkeit von Texten in Einklang bringen sollen (vgl. etwa Bundesverwaltungsamt, 2002; Hellinger \& Bierbach, 1993).

Die häufig anzutreffende Unzufriedenheit mit der ,Lesbarkeit' von geschlechtergleichbehandelnden Schreibweisen lässt sich auf mindestens zwei Ursachenkomplexe zurückführen. Zum einen sind aufgrund der jahrhundertelangen Tradition des generischen Maskulinums in praktisch allen Textsorten neue Schreibweisen, zumal solche, die andere Wortanmutungen hervorbringen, wie etwa das Schrägstrich-Prinzip („Die Beamt/inn/en“), ungewohnt. Lesen ist eine hochgradig automatisierte Tätigkeit (Logan, 1997), die auf der kognitiv aufwandsarmen Wiedererkennung von Zeichenkonfigurationen beruht. Ungewohnte Schreibweisen erschweren oder verhindern die automatische Verarbeitung von Wörtern, erhöhen daher kurzfristig den kognitiven Leseaufwand und unterbrechen damit den ,Lesefluss'. Genau darin liegt zwar der genderbezogene intendierte Effekt (nämlich die eingeschliffenen Verarbeitungsroutinen mit der expliziten Geschlechtergleichbehandlung zu konfrontieren und damit neue genderbezogene Denkmuster zu aktivieren); aus leseökonomischer Perspektive ergibt sich aber in der 
Tat eine objektive Steigerung der Verarbeitungsanforderungen: Die für die Lektüre zu investierende Denkleistung ist höher, was sich auch in einer Steigerung der für das Lesen benötigten Zeit niederschlagen kann. Beides - Aufwand und Zeiteinsatz - wird in vielen Lesekontexten, zumal bei der Lektüre journalistischer Texte, negativ bewertet.

Zum anderen konfligieren geschlechtergleichbehandelnde Schreibweisen gerade in leseaffinen Kontexten (etwa im Bereich des Qualitätsjournalismus, des Literaturbetriebs, des Schul- und Hochschulwesens) mit textästhetischen Präferenzen. Metaphern wie „Wortverstümmelung“ bringen das Unbehagen von Literaten mit den neuen Schreibweisen zum Ausdruck. „Placing opposition to feminist linguistic change [is] based on appeals to some „esthetic" criterion ... Dismissing attempts to avoid sexist language has been and continues to be the case in prescriptive handbooks, as "clumsy“, "wordy“ and „awkward“ is a way of obstructing efforts to include women in language use” (Penelope, 1982, S. 844). Für Literaturexpert/inn/en und Publizist/inn/en mit ausgeprägtem Gespür für die Feinheiten der Sprachgestaltung geht es entsprechend weniger um die Inkompatibilität der neuen Schreibweisen mit ihren Leseautomatismen (Lesbarkeit im technischen Sinne), sondern um eine Beeinträchtigung der Qualität von Texten als (Kunst-)Werk, unabhängig von der Textfunktion, gesellschaftliche Realität abzubilden. Aus beiden Argumentationslinien lässt sich jedoch die Vermutung ableiten, dass Texte, die geschlechtergleichbehandelnde Schreibweisen verwenden, hinsichtlich ihrer sprachlichen und journalistischen Qualität schlechter bewertet werden dürften als Texte, die die vertraute Form, namentlich das generische Maskulinum, verwenden.

\subsection{Zusammenfassung und forschungsleitende Annabmen}

Die bisherigen Ausführungen verweisen darauf, dass die Art der Geschlechterrepräsentation in Medienberichten Effekte auf die Verarbeitung und Bewertung der Botschaft durch das Publikum ausübt. Formulierungen, die explizit beide Geschlechter nennen, wenn Personen(gruppen) bezeichnet werden, sollten den gedanklichen Einbezug beider Geschlechter in den Situationsmodellen der Leser/innen fördern, also im Vergleich zu der traditionellen Form des generischen Maskulinums die Berücksichtigung von weiblichen Personen steigern, wobei dieser Effekt davon profitieren dürfte, dass die Nennung beider Geschlechter konsequent an allen relevanten Stellen eines Artikels vorgenommen wird. In ähnlicher Weise sollte die genderbezogene Zusammenstellung von Fallbeispielen, die zur Illustration des berichteten Sachverhalts präsentiert werden, auf den gedanklichen Einbezug von Frauen wirken (vgl. oben: 2.2). Diesen im Sinne der Geschlechtergleichbehandlung wünschbaren Effekten stehen - so die Annahme - negative Bewertungen der sprachlichen und journalistischen Textqualität gegenüber, weil gleichbehandelnde Formulierungen den Leseaufwand (mithin die Lesezeit) erhöhen und als textästhetisch suboptimal eingeschätzt werden.

\section{Experiment 1: Einfluss der sprachlichen Geschlechterrepräsentation auf den gedanklichen Einbezug von Frauen und die Textbewertung}

\subsection{Problemstellung und Hypothesen}

Das Experiment wurde mit dem Ziel durchgeführt, mögliche Unterschiede im gedanklichen Einbezug von Frauen bei variierender sprachlicher Geschlechterrepräsentation zu prüfen. Anhand zweier Versionen eines Textes, bei denen die sprachliche Geschlechterrepräsentation manipuliert wurde, sollten die Teilnehmer/innen der Studie den Frauen- 
und Männeranteil an der im Text dargestellten Gruppe schätzen. Ausgehend von den in Abschnitt (2.) dargestellten Überlegungen wurde folgende Hypothese aufgestellt:

H1. Leser/innen eines Zeitungsartikels mit gleichberechtigter sprachlicher Geschlechterdarstellung schätzen den Anteil von weiblichen Beteiligten an dem im Artikel berichteten Ereignis höher ein als Leser/innen eines inhaltlich identischen Artikels, in dem das generische Maskulinum verwendet wird.

Durch die veränderte Präsentation der Geschlechterformen im Text wird weiterhin eine Verschlechterung der Textbewertung im Hinblick auf die Lesbarkeit und die journalistisch-sprachliche Qualität des Texts als plausibel erachtet. In diesem Zusammenhang wird auch ein verlängernder Effekt der Nennung beider Geschlechter auf die Lesedauer angenommen. Dementsprechend ergeben sich zwei weitere Hypothesen:

H2. Das Publikumsurteil über die journalistisch-sprachliche Qualität eines Zeitungsartikels mit gleichberechtigter sprachlicher Geschlechterdarstellung fällt negativer aus als bei einem inhaltlich identischen Artikel, in dem das generische Maskulinum verwendet wird.

H3. Die Lesedauer eines Zeitungsartikels mit gleichberechtigter Geschlechterdarstellung verlängert sich im Vergleich zu einem inhaltlich identischen Artikel, in dem das generische Maskulinum verwendet wird.

\subsection{Methode}

\subsubsection{Operationalisierung der unabhängigen Variablen}

Zum Test der Hypothesen wurde ein Experiment durchgeführt, das sich an das Forschungsdesign von Braun et al. (1998) anlehnt. Anders als bei dieser und anderen früheren Studien (mit Ausnahme von Rothmund \& Christmann, 2002) wurde hier jedoch ein längerer Artikel (im Durchschnitt 445 Wörter) verwendet, so dass an 23 Stellen im Text eine Variation der Geschlechterform möglich war. Thematisch betraf der Artikel einen Ärztestreik und damit einen mehr oder weniger geschlechtsneutralen Kontext (laut Statistischem Bundesamt liegt der Anteil der Frauen im Ärzteberuf bei knapp 40 Prozent). Als gleichberechtigte Form der Geschlechterrepräsentation wurde die SchrägstrichSchreibweise verwendet, da diese die Gleichbehandlung der Geschlechter besonders explizit auszudrücken vermag und zugleich eine platzsparende Variante (etwa im Vergleich zur jeweils ausgeschriebenen Doppelnennung wie z. B. „Beamtinnen und Beamte") darstellt. Damit verbunden ist indes eine erwartbar starke kognitive Mehrbelastung für die Leser/innen wegen der Abweichung von der konventionellen Wortgestaltung ohne Schrägstriche.

Der Text ist angelehnt an einen Artikel aus der Online-Ausgabe der Süddeutschen Zeitung vom 29.11.2005. Er wurde gekürzt, teilweise modifiziert und den jeweiligen experimentellen Bedingungen in Bezug auf die sprachliche Repräsentation der Geschlechter angepasst. In der ersten Version wurden sowohl maskuline als auch feminine Formen gleichberechtigt eingesetzt (Version „gleichberechtigt“). Die zweite Version enthielt ausschließlich das generische Maskulinum (Version „generisches Maskulinum“). Die Bezeichnungen von Personen beiderlei Geschlechts wurden in diesem Fall durch die maskuline Form ausgedrückt (die Ärzte, die Studenten usw.).

An insgesamt 23 Stellen im Text der ersten Version wurden die gleichberechtigten Formen durch die Schrägstrich-Schreibweise präsentiert (die Beamt/inn/en, die Ärzt/ inn/e/n). Hier muss beachtet werden, dass sich der Wortstamm durch die Ableitung der femininen Form in einigen Fällen verändert. So löst die Endung auf -in im Singular des 
Wortes Arzt einen Umlaut im Wortstamm aus (der Arzt - die Ärztin). Dieser Sonderfall kann nicht in der Schrägstrich-Schreibweise dargestellt werden, so dass in diesen Fällen beide Formen in einer separaten Nennung präsentiert wurden („Der Gedanke, nachts in der Notaufnahme auf einen Arzt/eine Ärztin zu treffen...“).

\subsubsection{Operationalisierung der abhängigen Variablen}

Die Messung des geschätzten Franenanteils erfolgte, indem die Versuchspersonen nach der Lektüre des Artikels darum gebeten wurden, den prozentualen Anteil der Frauen bei dem im Text beschriebenen Ereignis einzuschätzen. Als Vergleichsgröße wurde zudem die Einschätzung des Frauenanteils in der Population, aus der die Teilnehmer der Demonstration stammen (die deutsche Ärzteschaft insgesamt), in Prozent erbeten. Damit sollte geprüft werden, inwiefern ein Effekt der Sprachgestaltung wie erwartet auf das berichtete Ereignis begrenzt ist oder aber in einer übergeordneten Kategorie auftritt. Zur Maskierung der zentralen Frage wurde zudem erhoben, welchen Anteil bestimmter Fachärzte an den Demonstrierenden die Versuchspersonen vermuteten.

Die journalistisch-sprachliche Qualität wurde anhand von sieben Items im Fragebogen erhoben (z. B. „Der Artikel war insgesamt gut lesbar“; alle Items sind im Anhang dokumentiert). Die Items wurden jeweils auf fünfstufigen Skalen („stimme überhaupt

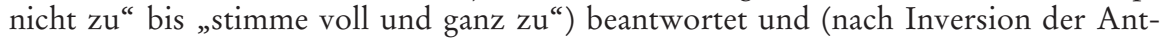
wortwerte für contrait-ausgerichtete Items) zu einem Index zusammengefasst (Cronbachs $\alpha=.81$ ). Die explizite Bewertung der Angemessenheit der Geschlechterdarstellung im Text wurde separat anhand des Einzel-Items „Die Art, wie das Geschlecht von Personen im Text wiedergegeben wurde, finde ich angemessen“ erfasst.

Die Lesedauer wurde gemessen, indem die Versuchspersonen gebeten wurden, jeweils unmittelbar vor und nach der Lektüre des Texts die aktuelle Uhrzeit auf den ausgehändigten Unterlagen einzutragen. Die Zeit wurde im Untersuchungsraum sekundengenau permanent auf eine Leinwand projiziert, so dass die Versuchspersonen jederzeit die genauen Werte erkennen und eintragen konnten. Die Lesedauer wurde entsprechend durch Subtraktion der angegebenen Anfangs- von der Enduhrzeit errechnet.

\subsubsection{Stichprobe und Durchführung}

Die Durchführung des Experiments erfolgte mit 41 studentischen Proband/inn/en in einem kommunikationswissenschaftlichen Seminar. Zur Maskierung des eigentlichen Untersuchungszwecks wurde die Bewertung von Zeitungsartikeln im Kontext des Ärztestreiks als Studienziel genannt. Eine befragte Person machte keine Angaben zu Alter und Geschlecht, wurde aber trotzdem in die Auswertung aufgenommen, da die restlichen Daten vollständig waren. Das Alter der Versuchspersonen lag zwischen 20 und 34 Jahren $(\mathrm{MW}=23,2, \mathrm{SD}=2,7), 14$ Versuchspersonen waren männlich, 26 weiblich.

Die Proband/inn/en erhielten ein sechsseitiges experimentelles Booklet. Auf der ersten Seite wurden sie gebeten, die aktuelle Uhrzeit einzutragen. Danach erfolgte die Lektüre des Zeitungsartikels. Die Zuweisung der Artikelversion wurde zufällig bei der Austeilung der Materialien vorgenommen, indem ein Stapel mit systematisch gemischten Textvarianten gebildet und dieser der Reihe nach an die anwesenden Teilnehmer ausgeteilt wurde. Unmittelbar nach der Lektüre wurden die Proband/inn/en erneut gebeten, die Uhrzeit einzutragen.

Im Anschluss erfolgten Fragen zur Schätzung des Frauen- und Männeranteils auf der Demonstration und der Verteilung der Geschlechter in der Gesamtärzteschaft (jeweils 
operationalisiert durch die offene Schätzung der Anteile in Prozent). Anschließend folgten die Items zur journalistisch-sprachlichen Qualität des Artikels. Zum Abschluss erfolgten Fragen zur Einstellung gegenüber der Debatte um Gleichberechtigung von Frauen und die Angabe von Alter und Geschlecht.

\subsection{Ergebnisse}

Wie Tabelle 1 zeigt, wird der Frauenanteil auf der Demonstration signifikant höher eingeschätzt, wenn eine gleichberechtigte Darstellungsweise gewählt wird. Hypothese H1 wird durch die Befunde mit einer relevanten Varianzaufklärung (14\%) gestützt. Auf die Einschätzung des Frauenanteils in der Ärzteschaft insgesamt hatte die Darstellungsweise hingegen keinen signifikanten Einfluss.

Tabelle 1: Einfluss der Geschlechterdarstellung auf die mentale Geschlechterrepräsentation der Versuchspersonen

\begin{tabular}{|c|c|c|c|c|c|c|}
\hline $\begin{array}{l}\text { Geschlechter- } \\
\text { repräsentation: } \\
\text { Schätzung des } \\
\text { prozentualen } \\
\text { Anteils von ... }\end{array}$ & $\begin{array}{l}\text { gleichberechtig- } \\
\text { te Schreibweise } \\
\text { MW } \\
\left(\mathrm{n}_{\max }=22\right)\end{array}$ & $\begin{array}{l}\text { generisches } \\
\text { Maskulinum } \\
\text { MW } \\
(\mathrm{n}=19)\end{array}$ & $\mathrm{F}$ & $\mathrm{df}$ & Signifikanz & $\eta^{2}$ \\
\hline $\begin{array}{l}\text { Frauen bei der } \\
\text { Demonstration. }\end{array}$ & $\begin{array}{c}40,1 \\
(\mathrm{SD}=9,9)\end{array}$ & $\begin{array}{c}33,7 \\
(\mathrm{SD}=5,5)\end{array}$ & 6,4 & $1 / 40$ & $\mathrm{p}<.05$ & .14 \\
\hline $\begin{array}{l}\text { Frauen innerhalb } \\
\text { der gesamten } \\
\text { deutschen Ärz- } \\
\text { teschaft. }\end{array}$ & $\begin{array}{c}39,0 \\
(\mathrm{SD}=11,0)\end{array}$ & $\begin{array}{c}36,8 \\
(\mathrm{SD}=7,5)\end{array}$ & 0,5 & $1 / 38$ & n.s. & .01 \\
\hline
\end{tabular}

$\mathrm{N}_{\text {max. }}=41, \mathrm{~N}_{\text {min. }}=39$

Zusätzlich wurde der Zusammenhang zwischen dem Geschlecht der Versuchspersonen und ihrer Einschätzung des Frauenanteils an der Demonstration und an der deutschen Ärzteschaft analysiert. Hier zeigte sich ein signifikanter Haupteffekt des Geschlechts der Befragten in Bezug auf den geschätzten Anteil von Frauen bei der Demonstration (Tabelle 2). Für die Schätzung des Anteils der Ärztinnen in der Gesamtbevölkerung in Abhängigkeit des Geschlechts der Befragten ergaben sich keine signifikanten Unterschiede.

Die Berechnung des Interaktionseffekts zwischen dem Geschlecht der Versuchsperson und der Geschlechterdarstellung ist an dieser Stelle aufgrund der geringen Fallzahlen in den einzelnen Gruppen nicht angezeigt.

Die gleichberechtigte Darstellung der Geschlechter im Artikel führte zu einer schlechteren Textbewertung. Tabelle 3 zeigt, dass der Bewertungsindex bei Leser/innen des Beitrags mit generischem Maskulinum einen signifikant höheren Mittelwert aufweist. Hypothese H2 wird demnach ebenfalls durch die Daten gestützt. Auch die Bewertung der Angemessenheit der Geschlechterdarstellung zeigt signifikante Unterschiede im Hinblick auf die unterschiedliche Geschlechterdarstellung. Diejenigen 
Befragten, die die gleichberechtigte Version lasen, beurteilten die Angemessenheit der Darstellung signifikant schlechter als diejenigen Befragten, die die Version mit generischem Maskulinum lasen.

Tabelle 2: Einfluss des Geschlechts der Versuchsteilnehmer/innen auf die mentale Geschlechterrepräsentation

\begin{tabular}{|c|c|c|c|c|c|c|}
\hline $\begin{array}{l}\text { Geschlechter- } \\
\text { repräsentation: } \\
\text { Schätzung des } \\
\text { prozentualen } \\
\text { Anteils von ... } \\
\end{array}$ & $\begin{array}{c}\text { Frauen } \\
\text { MW } \\
\left(\mathrm{n}_{\max }=26\right)\end{array}$ & $\begin{array}{l}\text { Männer } \\
\text { MW } \\
(\mathrm{n}=14)\end{array}$ & F & df & Signifikanz & $\eta^{2}$ \\
\hline $\begin{array}{l}\text { Frauen bei der } \\
\text { Demonstration. }\end{array}$ & $\begin{array}{c}35,3 \\
(\mathrm{SD}=9,0)\end{array}$ & $\begin{array}{c}41,1 \\
(\mathrm{SD}=8,0)\end{array}$ & 4,3 & $1 / 39$ & $\mathrm{p}<.05$ & .10 \\
\hline $\begin{array}{l}\text { Frauen inner- } \\
\text { halb der gesam- } \\
\text { ten deutschen } \\
\text { Ärzteschaft. }\end{array}$ & $\begin{array}{c}39,4 \\
(\mathrm{SD}= \\
10,1)\end{array}$ & $\begin{array}{c}35,3 \\
(\mathrm{SD}=7,4)\end{array}$ & 1,8 & $1 / 38$ & n.s. & .05 \\
\hline
\end{tabular}

$\mathrm{N}_{\text {max. }}=40, \mathrm{~N}_{\text {min. }}=39$

Tabelle 3: Einfluss der Geschlechterdarstellung auf die Bewertung der journalistischsprachlichen Qualität

\begin{tabular}{lcccccc}
\hline & $\begin{array}{c}\text { gleichberechtigte } \\
\text { Schreibweise } \\
\mathrm{MW} \\
(\mathrm{n}=20)\end{array}$ & $\begin{array}{c}\text { generisches } \\
\text { Maskulinum } \\
\mathrm{MW} \\
(\mathrm{n}=19)\end{array}$ & $\mathrm{F}$ & df & Signifikanz & $\eta^{2}$ \\
\hline $\begin{array}{l}\text { Journalistisch- } \\
\text { sprachliche Qualität } \\
\text { (Index aus 7 Items) }\end{array}$ & $\begin{array}{c}3,2 \\
(\mathrm{SD}=, 9)\end{array}$ & $\begin{array}{c}3,8 \\
(\mathrm{SD}=, 5)\end{array}$ & 5,7 & $1 / 38$ & $\mathrm{p}<.05$ & .13 \\
$\begin{array}{l}\text { Angemessenheit der } \\
\begin{array}{l}\text { Geschlechterdar- } \\
\text { stellung (Einzel- }\end{array}\end{array}$ & $\begin{array}{l}1,8 \\
\text { Item) }\end{array}$ & $\begin{array}{c}\text { (SD }=1,1) \\
(\mathrm{SD}=1,1)\end{array}$ & 8,5 & $1 / 38$ & $\mathrm{p}<.05$ & .18 \\
\hline
\end{tabular}

$\mathrm{N}=39$; Skala: 1 = trifft gar nicht $\mathrm{zu}, 5=$ trifft voll und ganz $\mathrm{zu}$

Die durchschnittliche Lesedauer der Artikel unterscheidet sich ebenfalls zwischen den experimentellen Bedingungen. Für den Artikel mit der ausschließlichen Verwendung des generischen Maskulinums benötigten die Teilnehmer im Durchschnitt 178,8 (SD $=30,3)$ Sekunden. Wurde die Schrägstrich-Schreibweise eingesetzt, erhöhte sich die durchschnittliche Lesezeit um 19,7 auf 198,5 (SD = 36,3) Sekunden. Dieser Unterschied bewegt sich inferenzstatistisch im Trendbereich $\left(\mathrm{F}(1 / 38)=3,4 ; \mathrm{p}<.08, \eta^{2}=.08\right)$ und spricht - mit geringen Einschränkungen in Bezug auf die Irrtumswahrscheinlichkeit für Hypothese $H 3$. 


\subsection{Diskussion}

Die Ergebnisse des Experiments stützen die theoretisch hergeleiteten Annahmen. Ein konsequenter Einsatz der Schrägstrich-Schreibweise erhöht den gedanklichen Einbezug von Frauen bei der mentalen Repräsentation des berichteten Ereignisses. Die Varianzaufklärung ist - ebenfalls wie aus der Textverstehensforschung ableitbar - relativ begrenzt, aber mit 14 Prozent auch alles andere als trivial. Mit diesem normativ wünschenswerten Ergebnis geht allerdings auch eine deutliche Verschlechterung der Textbewertung einher. Die Daten zur benötigten Lesezeit lassen vermuten, dass sich dieses Ergebnis teilweise auf den erhöhten Leseaufwand zurückführen lässt. Interessant ist der Unterschied zwischen männlichen und weiblichen Versuchspersonen hinsichtlich des gedanklichen Einbezugs von Frauen: Männer berücksichtigen Frauen in ihren mentalen Repräsentationen der berichteten Demonstration - über beide Artikelversionen hinweg - stärker als Frauen. Die deskriptiven Befunde verweisen auf einen möglichen Interaktionseffekt zwischen Geschlechterdarstellung im Text und Geschlecht der Versuchsperson: Möglicherweise reagieren Männer deutlich stärker auf die Art der sprachlichen Geschlechterdarstellung als Frauen. Die Stichprobengröße der Studie erlaubt hier aber keine belastbare entsprechende Schlussfolgerung. Folgestudien sollten diesem Phänomen nachgehen.

\section{Studie 2: Replikation von Studie 1 und Erweiterung um den Aspekt der Geschlechterrepräsentation in Fallbeispielen}

\subsection{Hypothesen}

Das Ziel der zweiten Studie war zum einen die Replikation des ersten Experiments. Zum anderen sollte nun auch der Einfluss geschlechterbezogen unterschiedlich zusammengesetzter Fallbeispiele in einem Artikel auf den gedanklichen Einbezug von Frauen getestet werden (vgl. oben: 2.2.). Analog zu dem durch die Literatur bereits gut belegten Phänomen der Übernahme aussagebezogener Realitätsvorstellungen aus Fallbeispielen gehen wir davon aus, dass auch eine Verallgemeinerung personenbezogener Merkmale von Fallbeispielurheber/inne/n auf die Grundgesamtheit, aus der sie stammen, von den Rezipient/inn/en vorgenommen wird. Dementsprechend sollte sich ein stärkerer gedanklicher Einbezug von Frauen feststellen lassen, wenn den Versuchspersonen geschlechterbezogen ausgewogene statt ausschließlich männliche Fallbeispiele präsentiert werden:

H4. Leser/innen eines Zeitungsartikels mit geschlechterbezogen ausgewogen zusammengestellten Fallbeispielen schätzen den Anteil weiblicher Beteiligter am berichteten Geschehen höher ein als Leser/innen eines inhaltlich identischen Artikels mit ausschließlich männlichen Fallbeispielen.

\subsection{Methode}

Das Experiment replizierte das Design von Studie 1, ergänzte aber zur Prüfung von H4 einen weiteren experimentellen Faktor: Zusätzlich zur Variation der sprachlichen Geschlechterdarstellung wurden Fallbeispiele in zwei unterschiedlichen Varianten in den Text eingeführt. Während in einer Version des Artikels zwei Ärzte stellvertretend für die Gesamtheit der Demonstrierenden ihre Meinung zur Gesundheitsreform in direkter Rede äußerten (Versuchsbedingung mit rein männlichen Fallbeispielen), waren 
es in der anderen Version jeweils eine Ärztin und ein Arzt (Versuchsbedingung mit geschlechterbezogen ausgewogenen Fallbeispielen). Der Inhalt der Aussagen blieb dabei identisch. Im Sinne der Fallbeispieldefinition von Daschmann (2001, S. 79) wurden die im Artikel dargestellten Aussagen nicht als persönliche Meinung einzelner Ärzte bzw. Ärztinnen, sondern als stellvertretend für die gesamte Gruppe der Ärzteschaft skizziert. Es ergab sich also ein 2 (sprachliche Geschlechterdarstellung) x 2 (geschlechterbezogene Fallbeispielkomposition)-Design.

Die abhängigen Variablen wurden exakt wie in Studie 1 erhoben (vgl. oben: 3.2.2), wobei der Index zur journalistisch-sprachlichen Qualität erneut eine zufriedenstellende Reliabilität (Cronbachs $\alpha=.81$ ) aufwies. Die Datenerhebung wurde im Rahmen einer kommunikationswissenschaftlichen Lehrveranstaltung durchgeführt. Bei den Versuchspersonen handelte es sich um 67 Studierende aus den Fachbereichen Journalismus, PR, Medienmanagement und Wirtschaftswissenschaft. Das Alter der 48 weiblichen und 19 männlichen Versuchspersonen bewegte sich zwischen 18 und 34 Jahren $(\mathrm{MW}=22,8$, $\mathrm{SD}=3,4)$. Die Maskierung des Untersuchungsinteresses und die Randomisierung der Versuchsbedingungen erfolgten analog zum bereits im Rahmen des ersten Experimentes geschilderten Vorgehen.

\subsection{Ergebnisse}

Personen, die die Artikelversion mit gleichberechtigter Schreibweise gelesen hatten, schätzten den Anteil weiblicher Demonstrierender rund drei Prozentpunkte höher ein als die Leser/innen der Artikelversion mit generischem Maskulinum (Tabelle 4). Allerdings konnte dieser erwartungskonforme Unterschied nicht gegen den Zufall abgesichert werden. Dieses Ergebnis entspricht also nur deskriptiv, nicht aber inferenzstatistisch dem in $H 1$ postulierten und in Studie 1 beobachteten Effekt. Auch trat in Studie 2 keine Wechselwirkung der Geschlechterdarstellung mit dem Geschlecht der Versuchspersonen auf $(\mathrm{F}<0.5)$. Hatte die Verwendung der gleichberechtigten Schreibweise in Experiment 1 nur eine marginale Wirkung auf Frauen, dafür aber eine umso stärkere Wirkung auf Männer, so zeigte sich im Rahmen der zweiten Studie eine gleichförmigere Beeinflussung der Versuchspersonen beider Geschlechter. Die angewandte Stimulusvariation führte bei Frauen in diesem Fall sogar zu geringfügig höheren Gruppenunterschieden als bei den Männern.

Mit Bezug auf $H 4$ zeigte sich, dass ein Gruppenunterschied hinsichtlich des geschätzten Frauenanteils an den Demonstrierenden zwischen den beiden Artikelversionen mit unterschiedlichen Fallbeispielanordnungen besteht. Die Verwendung der ausgewogenen Fallbeispielanordnung, in der jeweils eine Ärztin und ein Arzt zu Wort kommen, führte gegenüber der Version, in der nur Ärzte auftreten, zu einer rund fünf Prozentpunkte höheren Einschätzung des Anteils weiblicher Demonstrierender. Inferenzstatistisch bewegt sich der Effekt im Trendbereich $\left[\mathrm{F}(1 / 66)=3.3 ; \mathrm{p}<.08 ;{ }^{2}=.05\right]$. Konform mit $\mathrm{H} 4$ zeigt sich also tendenziell, dass das Geschlecht der als Fallbeispiele präsentierten Personen von den Versuchspersonen auf die dahinterstehende Personengruppe verallgemeinert wird. Dies trifft indes nicht auf die Einschätzung der deutschen Ärzteschaft insgesamt zu.

Auch hinsichtlich der Beurteilung der journalistisch-sprachlichen Qualität des Artikels (H2) zeigt sich ein ähnlicher Befund wie schon im ersten Experiment: Die Artikelversionen, in denen die gleichberechtigte Darstellungsweise der Geschlechter enthalten war, wurden schlechter beurteilt (Tabelle 5). Der Unterschied bewegt sich inferenzsta- 
tistisch im Trendbereich. Wechselwirkungen mit dem Geschlecht der Versuchspersonen zeigten sich bezüglich der Artikelbewertungen nicht $(\mathrm{F}<0.2)$.

Wie schon in der ersten Studie, so wurde auch in Experiment 2 die Angemessenheit der Geschlechterdarstellung im Artikel von jenen Proband/inn/en signifikant positiver eingestuft, die die Artikelversionen mit generischem Maskulinum gelesen haben (Tabelle 5). Eine Wechselwirkung mit dem Geschlecht besteht nicht $(\mathrm{F}<.02)$.

\section{Tabelle 4: Einfluss von Geschlechterdarstellung und Fallbeispielkomposition auf die} mentale Geschlechterrepräsentation der Versuchspersonen

\begin{tabular}{|c|c|c|c|c|}
\hline & \multicolumn{2}{|c|}{ Faktor 1: Geschlechterdarstellung } & \multicolumn{2}{|c|}{ Faktor 2: Fallbeispielkomposition } \\
\hline $\begin{array}{l}\text { Geschlechter- } \\
\text { repräsentation } \\
\text { Schätzung des } \\
\text { prozentualen } \\
\text { Anteils von ... } \\
\end{array}$ & $\begin{array}{c}\text { gleichberechtigte } \\
\text { Schreibweise } \\
\text { MW } \\
(\mathrm{n}=35)\end{array}$ & $\begin{array}{c}\text { generisches } \\
\text { Maskulinum } \\
\text { MW } \\
\left(\mathrm{n}_{\max }=32, \mathrm{n}_{\min }=31\right)\end{array}$ & $\begin{array}{c}\text { Fallbeispiele } \\
\text { einer Ärztin und } \\
\text { eines Arztes } \\
\text { MW } \\
(\mathrm{n}=35)\end{array}$ & $\begin{array}{c}\text { Fallbeispiele } \\
\text { zweier Ärzte } \\
\text { MW } \\
\left(\mathrm{n}_{\max }=32, \mathrm{n}_{\min }=31\right)\end{array}$ \\
\hline $\begin{array}{l}\text { Frauen bei der } \\
\text { Demonstration.a }\end{array}$ & $\begin{array}{c}42,3 \\
\operatorname{SD}(11,7)\end{array}$ & $\begin{array}{c}39,0 \\
\operatorname{SD}(11,2)\end{array}$ & $\begin{array}{c}43,2 \\
\operatorname{SD}(11,6)\end{array}$ & $\begin{array}{c}38,0 \\
\operatorname{SD}(10,9)\end{array}$ \\
\hline $\begin{array}{l}\text { Frauen inner- } \\
\text { halb der gesam- } \\
\text { ten deutschen } \\
\text { Ärzteschaft.b }\end{array}$ & $\begin{array}{c}39,6 \\
\operatorname{SD}(8,3)\end{array}$ & $\begin{array}{c}38,1 \\
\operatorname{SD}(10,8)\end{array}$ & $\begin{array}{c}38,7 \\
\operatorname{SD}(9,4)\end{array}$ & $\begin{array}{c}39,2 \\
\operatorname{SD}(9,4)\end{array}$ \\
\hline
\end{tabular}

a Haupteffekt der Geschlechterdarstellung: $\mathrm{F}(1,66)=1.3 ;$ n.s., $\eta^{2}=.02$

a Haupteffekt Fallbeispielkomposition: $\mathrm{F}(1,66)=3.3 ; \mathrm{p}<.08, \eta^{2}=.05$

b Haupteffekt Geschlechterdarstellung: $\mathrm{F}(1,65)=0.4 ;$ n.s., $\eta^{2}=.01$

b Haupteffekt Fallbeispielkomposition: $\mathrm{F}(1,65)=0.1 ;$ n.s., $\eta^{2}=.00$

Tabelle 5: Einfluss der Geschlechterdarstellung im Artikel auf Lesedauer und subjektiv eingeschätzte sprachlich-journalistische Qualität des Artikels

\begin{tabular}{|c|c|c|c|c|c|c|}
\hline Lesbarkeit des Artikels & $\begin{array}{l}\text { gleichberech- } \\
\text { tigte Darstel- } \\
\text { lungsweise }\end{array}$ & $\begin{array}{l}\text { generisches } \\
\text { Maskulinum }\end{array}$ & $\mathrm{df}$ & $\mathrm{F}$ & Signifikanz & $\eta^{2}$ \\
\hline $\begin{array}{l}\text { Index: sprachlich- } \\
\text { journalistische Qualität } \\
\text { des Artikels (Index auf } \\
7 \text { Items) }\end{array}$ & $\begin{array}{c}3,6 \\
(\mathrm{SD}=0,7)\end{array}$ & $\begin{array}{c}3,8 \\
(\mathrm{SD}=0,5)\end{array}$ & $1 / 63$ & 3.35 & $\mathrm{p}<.08$ & .05 \\
\hline $\begin{array}{l}\text { Angemessenheit der } \\
\text { Geschlechterdarstellung } \\
\text { (Einzel-Item) }\end{array}$ & $\begin{array}{c}2,6 \\
(\mathrm{SD}=1,2)\end{array}$ & $\begin{array}{c}3,2 \\
(\mathrm{SD}=0,9)\end{array}$ & $1 / 66$ & 5.1 & $\mathrm{p}<.05$ & .08 \\
\hline
\end{tabular}

Auch zeigte sich bezüglich $H 3$, dass die Versuchspersonen zum Lesen der Artikel mit gleichberechtigter sprachlicher Darstellungsweise der Geschlechter $(\mathrm{M}=196 \mathrm{sec}, \mathrm{SD}$ $=51 \mathrm{sec}$ ) deutlich länger brauchten als für die Versionen mit generischem Maskulinum $(\mathrm{M}=178 \mathrm{sec}, \mathrm{SD}=36 \mathrm{sec})$. Inferenzstatistisch erweist sich der Unterschied als allenfalls marginal bedeutsam $\left(\mathrm{F}(1 / 67)=2.8 ; \mathrm{p}<.11 ; \eta^{2}=.04\right)$. Andererseits stimmt der gefundene Effekt mit den Ergebnissen der ersten Teilstudie überein. Die Verwendung einer 
gleichberechtigten Darstellungsweise innerhalb journalistischer Artikel führt demnach tendenziell zu einer Erhöhung der von den Rezipienten benötigten Lesezeit.

\subsection{Diskussion}

Die Befunde der ersten Studie konnten teilweise repliziert werden, wobei die Absicherung der in $H 1$ bis $H 3$ postulierten Effekte gegen den Zufall nur teilweise gelungen ist. Im Hinblick auf den gedanklichen Einbezug von Frauen könnte der hinzugekommene und hypothesenkonforme Effekt der Fallbeispielkomposition (H4) interferiert haben, also die Wirkung der sprachlichen Geschlechterrepräsentation überdeckt haben. So bleibt festzuhalten, dass die Geschlechterzusammensetzung der Fallbeispiele Folgen für die genderbezogene mentale Repräsentation berichteter Sachverhalte durch Nachrichtenrezipient/inn/en besitzt. Wie schon in der ersten Studie, so beziehen sich die Einflüsse der Artikelgestaltung (in Studie 1: der Sprachgestaltung; in Studie 2: der Sprach-, vor allem aber der Fallbeispielgestaltung) auf das berichtete Ereignis und nicht auf eine übergeordnete Populationskategorie (die Ärzteschaft insgesamt). Auch dieser Befund ist plausibel, weil er auf die kurzfristigen, direkt-textbezogenen mentalen Repräsentationen zielt; übergeordnete Kategorien dürften erst durch wiederholte Konfrontation mit gleichbehandelnden Geschlechterdarstellungen tangiert werden (vgl. oben: 2.1). Der in Studie 1 noch vermutete Interaktionseffekt, wonach Rezipienten möglicherweise eher auf die genderbezogene Artikelausgestaltung reagieren als Rezipientinnen, konnte in Studie 2 nicht gefunden werden.

\section{Gesamtdiskussion}

Die beiden vorgestellten Experimente zeigen, dass die Ausgestaltung der Geschlechterrepräsentation in journalistischen Texten die angenommenen Effekte zeitigt. Sowohl die sprachliche als auch die fallbeispielbezogene Gleichbehandlung der Geschlechter in einem journalistischen Text besitzen das Potenzial, den gedanklichen Einbezug von Frauen während der Textverarbeitung zu steigern. Deskriptiv bewegen sich diese Wirkungen im Bereich weniger Prozentpunkte. Es gelingt also nicht, die inhaltliche Vorstellung vom berichteten Ereignis (hier: einer Demonstration) allein durch diese Maßnahmen dramatisch zu verändern. Mit Blick auf die Effektstärken (vgl. Tabellen 1 und 4) ist die Relevanz der Maßnahmen jedoch durchaus erkennbar: Wenn Autor/inn/en die Geschlechtergleichbehandlung ernst nehmen, können sie damit einen relevanten Einfluss auf ihr Publikum erreichen. Somit konvergieren die Befunde der vorgestellten Studien mit den Ergebnissen aus der Sprachpsychologie (z. B. Stahlberg \& Sczesny, 2001; vgl. oben: 2.1). Sie zeigen die Relevanz sprachlicher Geschlechtergleichbehandlung im Kontext der Rezeption von (Print-)Nachrichten am Beispiel eines für die tagesaktuelle Berichterstattung typischen Ereignisses mit Beteiligung vieler Personen, nämlich einer Demonstration.

Ungefähr mit gleicher, teils noch größerer Effektstärke sind auch die angenommenen ungünstigen Effekte der sprachlichen Geschlechtergleichbehandlung auf die Textbewertung aufgetreten. Die tendenziell längeren Lesezeiten für die mit Schrägstrich-Schreibweise ausgeführten Artikelversionen beider Studien deuten darauf hin, dass diese negative Bewertung wie erwartet in Teilen auf den erhöhten Verarbeitungsaufwand bei der Lektüre zurückgeht. Die Rezeption von 23 mit Schrägstrichen, zerlegten' Wörtern hat offenbar einen messbaren Unwillen bei den Versuchspersonen ausgelöst. Dieser Befund deckt sich mit den von Parks und Roberton (1998) berichteten negativen Einstellun- 
gen zur „non-sexist language“. Hier ist indes zu bedenken, dass eine weit verbreitete Anwendung geschlechtergleichbehandelnder Schreibweisen (etwa eine dauerhafte Implementation entsprechender Schreibregeln bei Tageszeitungen und Zeitschriften) in relativ kurzer Zeit zur Anpassung der automatischen Leseroutinen führen müsste. Die wiedererkennende Verarbeitung etwa von Schrägstrich-Schreibweisen könnte dadurch ebenfalls automatisiert werden und in der Folge deutlich weniger Verarbeitungsaufwand und -zeit konsumieren. Dass ein einzelner, ungewohnt gestalteter und deswegen ,sperriger' Text negativ(er) bewertet wird, muss also nicht zwangsläufig eine dauerhaft negative Publikumsreaktion auf solche Schreibweisen implizieren. Inwiefern solche Automatisierungs- und Gewöhnungseffekte tatsächlich eintreten und die wiederholte, routinemäßige Verarbeitung gleichbehandelnder Schreibweisen nicht mehr zu einer negativen Texteinschätzung führt, ist demnach eine wichtige Fragestellung für die weitere thematische Forschung.

Besondere Beachtung verdient auch der in beiden Experimenten aufgetretene Effekt, wonach das generische Maskulinum bei der expliziten Frage nach der Angemessenheit der Geschlechterrepräsentation im Artikel besser bewertet wurde als die Nennung beider Geschlechter. Dieser Befund könnte ebenfalls eine gewohnheitsbedingte Präferenz für die rein männliche Form reflektieren, aber auch ein Hinweis auf Reaktanz gegenüber explizit ,politisch korrekten' Neuerungen in der öffentlichen Kommunikation sein (Parks \& Roberton, 1998). Im letztgenannten Fall würde die Gewöhnung an die neue Schreibweise im Sinne einer Leseautomatisierung nicht zu besseren Bewertungen der journalistischen Qualität von Artikeln mit der neuen Schreibweise führen.

Im Zuge der Prüfung von Gewöhnungseffekten an geschlechtergleichbehandelnde Schreibweisen ist jedoch auch kritisch zu betrachten, inwiefern der in den vorgestellten Experimenten gefundene Effekt des verbesserten gedanklichen Einbezugs von Frauen durch die neue Schreibweise gerade durch die Automatisierung des Verarbeitens von Wörtern in dieser Schreibweise wieder nivelliert wird. Denn mit der Automatisierung könnte auch eine komplexitätsreduzierende mentale Repräsentation des von einem Wort (etwa „Beamt/inn/en“) bezeichneten Personenkreises einhergehen, die dann möglicherweise doch wieder männlich dominiert wird (etwa aufgrund der nachwirkenden Heuristiken aus der Tradition des generischen Maskulinums, vgl. Prentice, 1994; oben: 2.2). Die hier berichteten Effekte könnten also Novitätseffekte sein: Gerade weil die Schrägstrich-Schreibweise ungewohnt war, könnten die Leser/innen darauf aufmerksam geworden sein und die Bedeutung der betroffenen Wörter intensiver als bei normaler Lektüre elaboriert haben. Falls mit der Leseautomatisierung von Wörtern dieser Schreibweise solche Prozesse der zusätzlichen Elaboration ausbleiben, würden mit steigender Gewöhnung an diese Schreibweise deren Effekte auf den gedanklichen Einbezug von Frauen wieder in Frage stehen. Entsprechend lohnt sich für nachfolgende Untersuchungen ein langfristiges Forschungsdesign, das Gewöhnungseffekte an die hier kurzfristig als effektiv nachgewiesene - neue genderbezogene Schreibweise sowohl hinsichtlich des gedanklichen Einbezugs beider Geschlechter im Situationsmodell der Leser/innen als auch hinsichtlich der Textbewertung im Zeitverlauf abbildet.

In Bezug auf den gedanklichen Einbezug der Geschlechter, die Textbewertung und speziell auf den Bewertungsaspekt der Textästhetik ergibt sich die Anschlussfragestellung, welchen Effekt das Geschlecht der Rezipierenden direkt oder interaktiv mit der sprachlichen Geschlechtsrepräsentation im Text bedingt. Die vorgestellten Experimente weisen zu geringe Fallzahlen und unzureichend ausbalancierte Geschlechterverhältnisse auf, um diesen Aspekt vollständig analysieren zu können. Die Arbeit von Braun et al. (1998) verweist indes darauf, dass erhebliche Geschlechterunterschiede zu erwarten 
sind. Neben der Betrachtung von Genderunterschieden auf Publikumsseite (als Überwindung einer methodischen Limitation der vorgestellten Studien) sollten Folgestudien auch prüfen, inwiefern unterschiedlich sprach-, literatur- und leseaffine Personengruppen (z. B. Feuilletonist/inn/en, Lehrer/innen) auf die neue Schreibweise reagieren. Damit könnten auch etwaige Spezifika studentischer Stichproben kompensiert werden, die in den vorliegenden Studien rekrutiert wurden: Möglicherweise stellen sich die experimentellen Effekte für andere Personengruppen stärker oder schwächer dar. Replikationsstudien sollten überdies auf eine Verbreiterung der Ergebnisse in Bezug auf Berichtsthemen und berichtete Personenkreise zielen. Die Arbeitsgruppe um Dagmar Stahlberg hat hier komplexe Interaktionen zwischen der Gendergebundenheit von Thematisierungskontexten und sprachlichen Geschlechterrepräsentationen nachgewiesen (vgl. oben: 2.1). Zudem sollten auch andere Formen der sprachlichen Geschlechtergleichbehandlung getestet werden. Eventuell ergibt sich nämlich für andere Varianten (etwa „BeamtInnen“) ein günstigeres Verhältnis aus erhöhtem gedanklichen Einbezug von Frauen und dem Ausmaß der (negativen) Textbewertung. Es besteht demnach eine Reihe von Aufgaben für Replikations- und Erweiterungsstudien zum Aspekt der sprachlichen Geschlechtergleichbehandlung. Sofern derartige Replikations- und Erweiterungsstudien die hier berichteten Ergebnisse erhärten, dürfte die Zeit gekommen sein, mit den empirischen Argumenten eine Diskussion über textästhetische Maßstäbe in den jeweiligen Domänen und Lesekulturbereichen zu stimulieren (vgl. auch Penelope, 1982).

Auch wenn Replikationsstudien zum in Studie 2 beobachteten Fallbeispieleffekt ebenfalls notwendig sind (vor allem mit Bezug auf die im vorliegenden Fall noch ungenügende Teststärke, nämlich die variierte Anzahl der Fallbeispiele sowie die Anzahl der Versuchspersonen), weisen die thematischen Befunde zu diesem Teilaspekt bereits jetzt deutlich darauf hin, dass Journalist/inn/en bei der Auswahl ihrer Fallbeispiele relevante Gestaltungsspielräume haben, um den gedanklichen Einbezug von Frauen bei ihrem Publikum zu unterstützen. Bei vielen Berichten, die ,fallbeispielaffin' sind (Berger, 2001), dürften ausreichend Personen beider Geschlechter für die Einbindung als Fallbeispiele zur Verfügung stehen. Eine ausgewogene Gender-Mischung kann in solchen Fällen wünschbare Effekte auf die gedankliche Berücksichtigung von Frauen haben und sollte daher zu einer journalistisch-handwerklichen Leitlinie entwickelt werden. Die Legitimation dieses Vorschlags ist indes nicht nur in der normativen Perspektive der Geschlechtergleichbehandlung in der Gesellschaft zu sehen; vielmehr geht es auch um die Maßgabe der Realitätsadäquanz von Medienberichten (z. B. Wunden, 1996). Denn in dem Maße, in dem Personen beiderlei Geschlechts an einem Ereignis beteiligt sind, sollten journalistische Darstellungen des Ereignisses eben diese Tatsache auch widerspiegeln. Studie 2 hat Hinweise darauf erbracht, dass Fallbeispiele nicht nur hinsichtlich der in ihnen enthaltenen Äußerungen und Meinungen relevant für das Textverständnis sind, sondern auch hinsichtlich der erkennbaren Personeneigenschaften ihrer Urheber, also im vorliegenden Fall des Geschlechts.

Der vorliegende Beitrag hat versucht, theoriegeleitet-empirische Forschung zu einem normativ aufgeladenen Diskurs zu erbringen. Die Ergebnisse rechtfertigen die Fortsetzung und Ausdehnung dieser Forschung, so dass die damit verbundenen Fragen nach der ,guten journalistischen Arbeitspraxis' mittelfristig mit Hilfe theoretisch-empirischer Argumente diskutiert werden können. Die Logik empirischer Sozialforschung an sich sowie im vorliegenden Fall auch die erkennbaren, aber begrenzten Effektstärken verweisen aber darauf, dass eine Instrumentalisierung der empirischen Befunde für eine (quasi-)ideologische Debattenführung (also beispielsweise eine radikal-feministische 
Position oder eine ignorant-reaktante Haltung gegen Geschlechtergleichbehandlung in Medien und Gesellschaft, vgl. Mucchi-Faina, 2005) unangemessen wäre. Vielmehr wäre es wünschenswert, wenn die Reflexion über den Umgang mit Geschlechterdarstellungen in Medienbotschaften nüchterner und verstärkt mit Blick auf systematische Forschung betrieben werden würde.

\section{Literatur}

Bate, B. (1978). Non-sexist language use in transition. Journal of Communication, 28 (4), 139-149.

Berger, C. (2001). Making it worse than it is: Quantitative depictions of threatening trends in the news. Journal of Communication, 51 (4), 655-677.

Blaubergs, M. (1980). An analysis of classic arguments against changing sexist language. Women's Studies International Quarterly, 3, 135-147.

Braun, F., Sczesny, S. \& Stahlberg, D. (2005). Cognitive effects of masculine generics in German: An overview of empirical findings. Communications, 30, 1-21.

Braun, F., Gottburgsen, A., Sczesny, S. \& Stahlberg, D. (1998). Können Geophysiker Frauen sein? Generische Personenbezeichnungen im Deutschen. Zeitschrift für germanistische Linguistik, 26 (3), 265-283.

Brosius, H.-B., Schweiger, W. \& Rossmann, C. (2000). Auf der Suche nach den Ursachen des Fallbeispieleffekts: Der Einfluß von Anzahl und Art der Urheber von Fallbeispielinformationen. Medienpsychologie, 12 (3), 153-173.

Bundesverwaltungsamt (2002). Sprachliche Gleichbehandlung von Frauen und Männern (BBB Merkblatt M19, 2. Aufl.). Köln: Bundesverwaltungsamt.

Carroll, J. B. (Hrsg.) (1956). Language, thought, and reality: Selected writings of Benjamin Lee Whorf. Cambridge, MA: MIT Press.

Daschmann, G. (2001). Der Einfluss des Fallbeispiel-Effekts auf Leserurteile. Experimentelle Untersuchungen zur Medienwirkung. Konstanz: UVK.

Döring, N. (2003). Männliche Formen. Aviso, o. Jg. (Nr. 33, Juni 2003), 28.

Ganahl, D. J., Prinzen, T. J. \& Netzley, S. B. (2003). A content analysis of prime time commercials: A contextual framework of gender representation. Sex Roles, 49 (9-10), 545-551.

Gibson, R. \& Zillmann, D. (1994). Exaggerated versus representative exemplification in news reports. Perception of issues and personal consequences. Communication Research, 21 (5), 603-624.

Hellinger, M. \& Bierbach, C. (1993). Eine Sprache für beide Geschlechter: Richtlinien für einen nicht-sexistischen Sprachgebrauch. Bonn: Deutsche UNESCO-Kommission.

Irmen, L. \& Kaczmarek, N. (2000). Beeinflusst das grammatische Geschlecht die Repräsentation von Personen in einem mentalen Modell? Ein Vergleich zwischen einer englischsprachigen und einer deutschsprachigen Stichprobe. Vortrag auf dem 42. Kongress der Deutschen Gesellschaft für Psychologie, 24.-28.09.2000, Jena.

Irmen, L. \& Köhncke, A. (1996). Zur Psychologie des „generischen“ Maskulinums. Sprache E Kognition, o. Jg. (15), 152-166.

Irmen, L. \& Linner, U. (2005). Die Repräsentation generisch maskuliner Personenbezeichnungen: Eine theoretische Integration bisheriger Befunde. Zeitschrift für Psychologie, 213, 167-175.

Irmen, L. \& Roßberg, N. (2004). Gender markedness of language: The impact of grammatical and nonlinguistic information on the mental representation of person information. Journal of Language and Social Psychology, 23, 272-307.

Kintsch, W. (1988). The role of knowledge in discourse comprehension: A construction-integration model. Psychological Review, 95 (2), 163-182.

Logan, G. (1997). Automaticity and reading: Perspectives from the instance theory of automatization. Reading and Writing Quarterly, 13 (2), 123-146.

Martyna, W. (1978). Use of the generic masculine. Journal of Communication, 28 (4), 131-138. 
Mucchi-Faina, A. (2005). Visible or influential? Language reforms and gender (in)equality. Social Science Information, 44 (1), 189-215.

Parks, J. B., \& Roberton, M. A. (1998). Contemporary arguments against nonsexist language: Blaubergs (1980) revisited. Sex Roles, 39, 445-461.

Parks, J. B. \& Roberton, M. A. (2000). Development and validation of an instrument to measure attitudes toward sexist / nonsexist language. Sex Roles, 42 (5-6), 415-438.

Penelope, J. (1982). Two essays on language and change I: Power and the opposition to feminist proposals for language change. College English, 44 (8), 840-848.

Prentice, D. A. (1994). Do language reforms change our way of thinking? Journal of Language and Social Psychology, 13, 3-19.

Purnell, S. E. (1978). Politically speaking, do women exist? Journal of Communication, 28 (4), 150-155.

Pusch, L. F. (1979). Der Mensch ist ein Gewohnheitstier, doch weiter kommt man ohne ihr. Linguistische Berichte, 63, 84-102.

Roskos-Ewoldsen, B., Davies, J. \& Roskos-Ewoldsen, D. R. (2004). Implications of the mental models approach for cultivation theory. Communications: The European Journal of Communication Research, 29 (3), 345-364.

Rothmund J. \& Christmann, U. (2002). Auf der Suche nach einem geschlechtergerechten Sprachgebrauch. Muttersprache, 112 (4), 115-135.

Scheele, B. \& Gauler, E. (1993). Wählen Wissenschaftler ihre Probleme anders aus als WissenschaftlerInnen? Das Genus-Sexus-Problem als paradigmatischer Fall der linguistischen Relativitätsthese. Sprache E Kognition, o. Jg. (12), 59-72.

Stahlberg, D. \& Sczesny, S. (2001). Effekte des generischen Maskulinums und alternativer Sprachformen auf den gedanklichen Einbezug von Frauen. Psychologische Rundschau, 52 (3), 131-140.

Trömel-Plötz, S. (1982). Franensprache: Sprache der Veränderung. Frankfurt/M.: Fischer.

Wunden, W. (Hrsg.) (1996). Wabrheit als Medienqualität. Frankfurt/M.: Gemeinschaftswerk der evangelischen Publizistik.

Zillmann, D. \& Brosius, H.-B. (Hrsg.) (2000). Exemplification in communication: The influence of case reports on the perception of issues. Mahwah, NJ: Lawrence Erlbaum Associates.

Zillmann, D., Perkins, J. W. \& Sundar, S. S. (1992). Impression-formation effects of printed news varying in descriptive precision and exemplifications. Medienpsychologie, 4 (3), 168-185.

Zwaan, R. A. \& Radvansky, G. A. (1998). Situation models in language comprehension and memory. Psychological Bulletin, 123 (2), 162-185.

\section{Anhang: Items zur Bewertung der sprachlich-journalistischen Textqualität}

Das Antwortformat für alle Items war fünfstufig skaliert von „1 (trifft gar nicht zu)“ bis „,5 (trifft voll und ganz $\mathrm{zu}$ )“

Ich fand den Artikel gut verständlich.

Der Artikel war insgesamt gut lesbar.

Einige Formulierungen waren holprig.

Die journalistische Qualität des Artikels ist gut.

Es war anstrengend, den Text zu lesen.

Die Lektüre hat mir Spaß gemacht.

An einigen Stellen kam mir der Artikel gestelzt vor. 